\title{
Irritated Pigmented Plaque on the Scalp
}

\author{
Venkata Anisha Guda, BS; Sara E. Chapman, MD; Justin P. Bandino, MD
}

\section{Eligible for 1 MOC SA Credit From the ABD}

This Dermatopathology Diagnosis in our print edition is eligible for 1 self-assessment credit for Maintenance of Certification from the American Board of Dermatology (ABD). After completing this activity, diplomates can visit the ABD website (http://www.abderm.org) to self-report the credits under the activity title "Cutis Dermatopathology Diagnosis." You may report the credit after each activity is completed or after accumulating multiple credits.

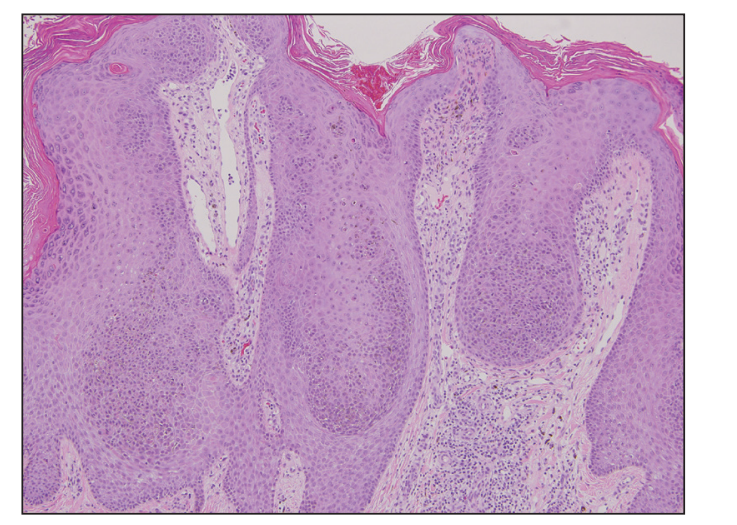

H\&E, original magnification $\times 100$.

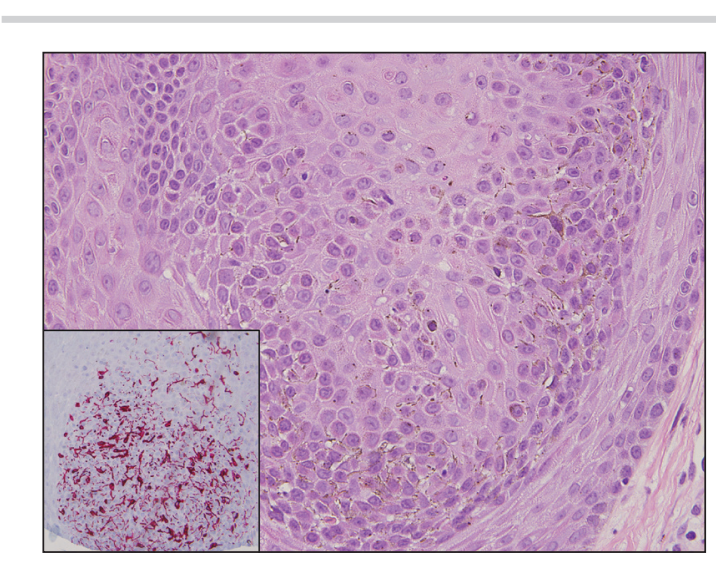

$\mathrm{H} \& \mathrm{E}$, original magnification $\times 400$ (MART-1, original magnification $\times 200$ [inset]).
A 49-year-old man with light brown skin and no history of skin cancer presented with a pruritic lesion on the scalp of 3 years' duration. Physical examination revealed a $7 \times 3-\mathrm{cm}$, brown, mammillated plaque on the left parietal scalp. A shave biopsy of the scalp lesion was performed.

\section{THE BEST DIAGNOSIS IS:}
a. clonal melanoacanthoma
b. hidroacanthoma simplex
c. malignant melanoma
d. seborrheic keratosis
e. squamous cell carcinoma in situ

PLEASE TURN TO PAGE 192 FOR THE DIAGNOSIS

\footnotetext{
Ms. Guda is from the UT Health San Antonio Long School of Medicine, Texas. Drs. Chapman and Bandino are from the Department of Dermatology, San Antonio Uniformed Services Health Education Consortium.

The authors report no conflict of interest.

The views expressed are those of the authors and do not reflect the official views or policy of the US Department of Defense.

Correspondence: Venkata Anisha Guda, BS (gudav@livemail.uthscsa.edu).

doi:10.12788/cutis.0092
} 


\section{THE DIAGNOSIS:}

\section{Clonal Melanoacanthoma}

M elanoacanthoma (MA) is an extremely rare, benign, epidermal tumor histologically characterized by keratinocytes and large, pigmented, dendritic melanocytes. These lesions are loosely related to seborrheic keratoses, and the term was first coined by Mishima and Pinkus ${ }^{1}$ in 1960. It is estimated that the lesion occurs in only 5 of 500,000 individuals and tends to occur in older, light-skinned individuals. ${ }^{2}$ The majority are slow growing and are present on the head, neck, or upper extremities; however, similar lesions also have been reported on the oral mucosa. ${ }^{3}$ Melanoacanthomas range in size from $2 \times 2$ to $15 \times 15 \mathrm{~cm}$; are clinically pigmented; and present as either a papule, plaque, nodule, or horn. ${ }^{2}$

Classic histologic findings of MA include papillomatosis, acanthosis, and hyperkeratosis with heavily pigmented dendritic melanocytes diffusely dispersed throughout all layers of the seborrheic keratosis-like epidermis. ${ }^{3}$ Other features include keratin-filled pseudocysts, Langerhans cells, reactive spindling of keratinocytes, and an inflammatory infiltrate. In our case, the classic histologic findings also were architecturally arranged in oval to round clones within the epidermis (quiz images 1 and 2). A MART-1 (melanoma antigen recognized by $\mathrm{T}$ cells) immunostain was obtained that highlighted the numerous but benign-appearing, dendritic melanocytes (quiz image 2 [inset]). A dual MART-1/Ki67 immunostain later was obtained and demonstrated a negligible proliferation index within the dendritic melanocytes. Therefore, the diagnosis of clonal MA was rendered. This formation of epidermal clones also is called the Borst-Jadassohn phenomenon, which rarely occurs in MAs. This subtype is important to recognize because the clonal pattern can more closely mimic malignant neoplasms such as melanoma.

Hidroacanthoma simplex is an intraepidermal variant of eccrine poroma. It is a rare entity that typically occurs in the extremities of women as a hyperkeratotic plaque. These typically clonal epidermal tumors may be heavily pigmented and rarely contain dendritic melanocytes; therefore, they may be confused with MA. However, classic histology will reveal an intraepidermal clonal proliferation of bland, monotonous, cuboidal cells with ample pink cytoplasm, as well as occasional cuticlelined ducts (Figure 1). ${ }^{4}$ These ducts will highlight with carcinoembryonic antigen and epithelial membrane antigen immunostaining.

Malignant melanoma typically presents as a growing pigmented lesion and therefore can clinically mimic MA. Histologically, MA could be confused with melanoma due to the increased number of melanocytes plus the appearance of pagetoid spread resulting from the diffuse presence of melanocytes throughout the neoplasm.
However, histologic assessment of melanoma should reveal cytologic atypia such as nuclear enlargement, hyperchromasia, molding, pleomorphism, and mitotic activity (Figure 2). Architectural atypia such as poor lateral circumscription of melanocytes, confluence and pagetoid spread of nondendritic atypical junctional melanocytes, production of pigment in deep dermal nests of melanocytes, and lack of maturation and dispersion of dermal melanocytes also should be seen. ${ }^{5}$ Unlike a melanocytic neoplasm, true melanocytic nests are not seen in MA, and the melanocytes are bland, normal-appearing but heavily pigmented, dendritic melanocytes. Electron microscopy has shown a defect in

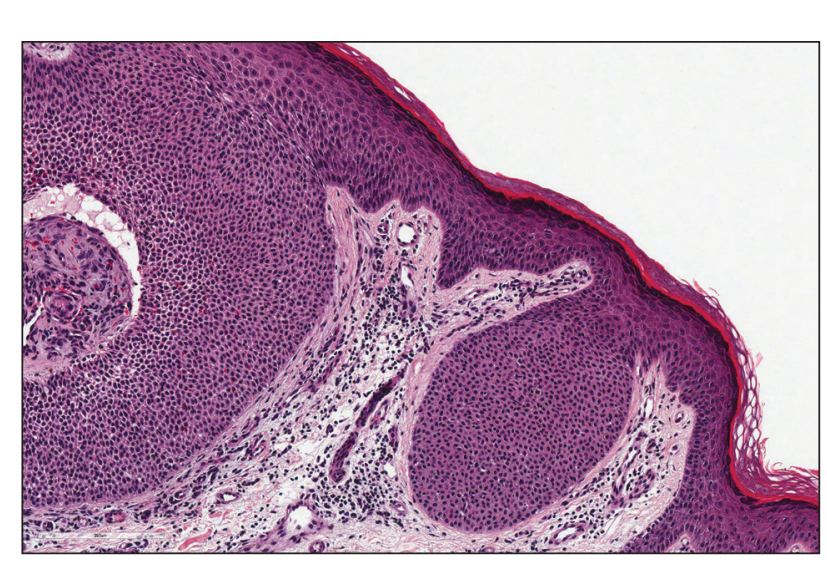

FIGURE 1. Hidroacanthoma simplex. Intraepidermal clonal proliferation of bland, monotonous, cuboidal cells with ample pink cytoplasm, as well as occasional cuticle-lined ducts (H\&E, original magnification $\times 200$ )

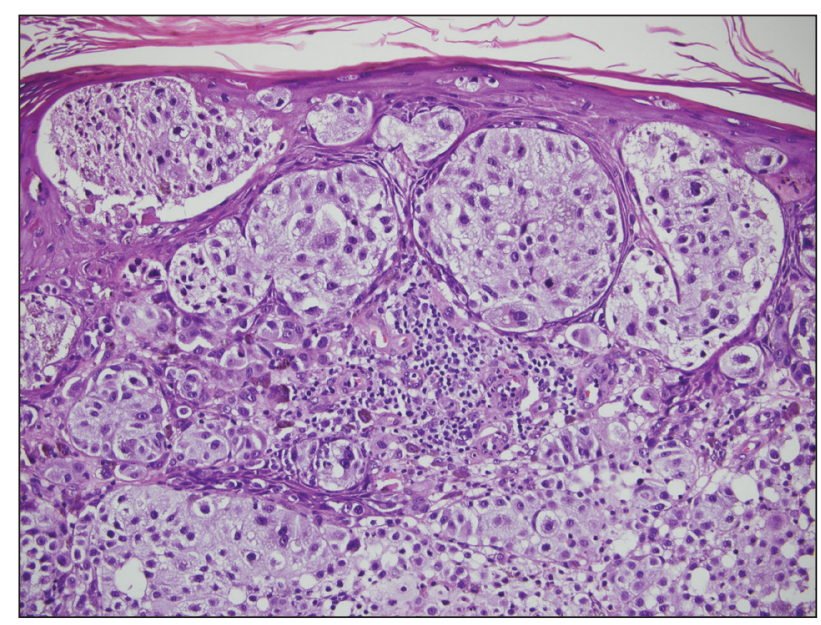

FIGURE 2. Melanoma. Large nests of variable sizes with cytologic atypia as well as confluent and pagetoid spread of junctional melanocytes $(\mathrm{H} \& \mathrm{E}$, original magnification $\times 200)$. 


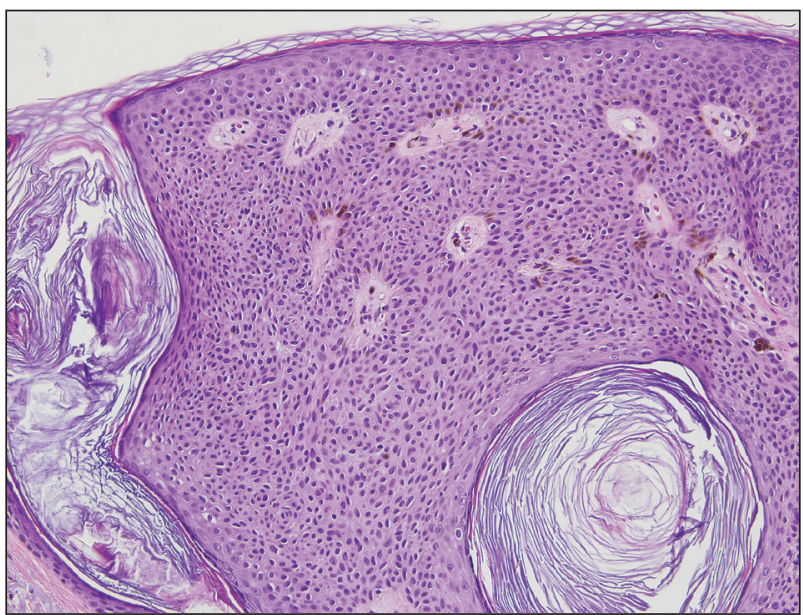

FIGURE 3. Seborrheic keratosis. Acanthotic epidermal growth of bland keratinocytes containing multiple cornified horn cysts and an overlying normal basketweave stratum corneum (H\&E, original magnification $\times 200$ ).

the transfer of melanin from these highly dendritic melanocytes to the keratinocytes. ${ }^{6}$

Similar to melanoma, seborrheic keratosis presents as a pigmented growing lesion; therefore, definitive diagnosis often is achieved via skin biopsy. Classic histologic findings include acanthotic or exophytic epidermal growth with a dome-shaped configuration containing multiple cornified hornlike cysts (Figure 3). ${ }^{7}$ Multiple keratin plugs and variably sized concentric keratin islands are common features. There may be varying degrees of melanin pigment deposition among the proliferating cells, and clonal formation may occur. Melanocyte-specific special stains and immunostains can be used to differentiate MA from seborrheic keratosis by highlighting numerous dendritic melanocytes diffusely spread throughout the epidermis in MA vs a normal distribution of occasional junctional melanocytes in seborrheic keratosis. ${ }^{2,8}$

Squamous cell carcinoma in situ presents histologically with cytologically atypical keratinocytes encompassing the full thickness of the epidermis and sometimes crushing the basement membrane zone (Figure 4). There is a loss of the granular layer and overlying parakeratosis that often spares the adnexal ostial epithelium. ${ }^{9}$ Clonal formation can occur as well as increased pigment production. In comparison, bland keratinocytes are seen in MA.

Establishing the diagnosis of MA based on clinical features alone can be difficult. Dermoscopy can prove to be

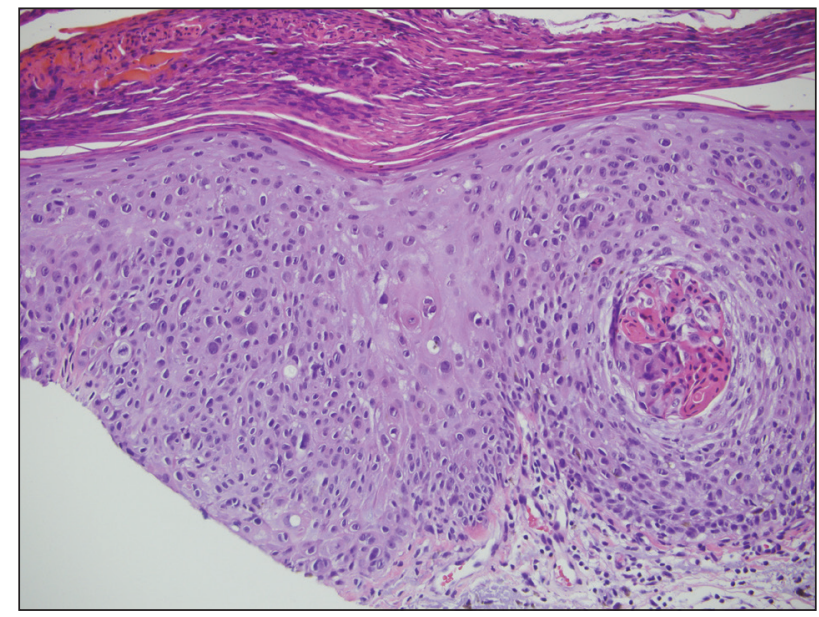

FIGURE 4. Squamous cell carcinoma in situ. Full-thickness epidermal atypia of cytologically atypical keratinocytes, crushing of the basement membrane zone, partial loss of the granular layer, and overlying parakeratosis $(H \& E$, original magnification $\times 200)$.

useful and typically will show a sunburst pattern with ridges and fissures. ${ }^{2}$ However, seborrheic keratoses and melanomas can have similar dermoscopic findings ${ }^{10}$; therefore, a biopsy often is necessary to establish the diagnosis.

\section{REFERENCES}

1. Mishima Y, Pinkus H. Benign mixed tumor of melanocytes and malpighian cells: melanoacanthoma: its relationship to Bloch's benign nonnevoid melanoepithelioma. Arch Dermatol. 1960;81:539-550.

2. Gutierrez N, Erickson C P, Calame A, et al. Melanoacanthoma masquerading as melanoma: case reports and literature review. Cureus. 2019;11:E4998.

3. Fornatora ML, Reich RF, Haber S, et al. Oral melanoacanthoma: a report of 10 cases, review of literature, and immunohistochemical analysis for HMB-45 reactivity. Am J Dermatopathol. 2003;25:12-15.

4. Rahbari H. Hidroacanthoma simplex-a review of 15 cases. Br J Dermatol. 1983;109:219-225

5. Smoller BR. Histologic criteria for diagnosing primary cutaneous malignant melanoma. Mod Pathol. 2006;19:S34-S40.

6. Mishra DK, Jakati S, Dave TV, et al. A rare pigmented lesion of the eyelid. Int J Trichol. 2019;11:167-169.

7. Greco MJ, Mahabadi N, Gossman W. Seborrheic keratosis. StatPearls. Treasure Island, FL: StatPearls Publishing; 2020. https://www.ncbi.nlm .nih.gov/books/NBK545285/. Accessed September 18, 2020.

8. Kihiczak G, Centurion SA, Schwartz RA, et al. Giant cutaneous melanoacanthoma. Int J Dermatol. 2004;43:936-937.

9. Morais P, Schettini A, Junior R. Pigmented squamous cell carcinoma: a case report and importance of differential diagnosis. An Bras Dermatol. 2018;93:96-98.

10. Chung E, Marqhoob A, Carrera C, et al. Clinical and dermoscopic features of cutaneous melanoacanthoma. JAMA Dermatol. 2015;151:1129-1130. 